\title{
A RARE VASCULITIS MIMICS: FIBROMUSCULAR DYSPLASIA
}

\author{
Catharine Luisa Rocha Soares ${ }^{1}$, Ian Jader Alves de Oliveira ${ }^{1}$, Oder Banhara Duarte ${ }^{1}$, Flavio Barboza $^{1, \star}$ \\ 1.Universidade Federal de Mato Grosso, Sinop (MT), Brazil. \\ *Corresponding author: flavio.fbarboza@uol.com.br
}

\section{BACKGROUND}

Fibromuscular dysplasia (FMD) is a rare, noninflammatory and nonatherosclerotic disease, which affects the muscles of medium and small caliber arteries. The FMD is most observed in women and the mainly affected arteries are the renal, cerebral, carotid (extracranial) and vertebral. The signs and symptoms depend on the compromised location, ranging from headaches and pulsatile tinnitus in cerebrovascular disease to resistant hypertension in renal involvement. The diagnosis consists of at least one focal or multifocal arterial lesion through examinations such as angioresonance, angiography or computed tomography angiography. Complications include aneurysms, stenosis and renovascular hypertension. Atherosclerosis and vasculitis are the main differential diagnoses of this disease.

\section{CASE REPORT}

A thin woman, 46 years old, with high blood pressure since she was 33, using two antihypertensive drugs. A long-distance runner, she developed high-intensity headache after a strenuous physical activity day. After that, she went to a neurologist who requested some tests: magnetic resonance imaging (MRI) showing 50\% stenosis in some segments of the right vertebral artery associated with its recent dissection (Fig. 1); cerebral arteriography that confirmed the MRI findings, excluding the possibility of a ruptured aneurysm. She was referred to the rheumatologist for investigation of possible vasculitis. The patient complained of a 40-days persistent headache even after suspension of physical activity. She denied fever, weight loss and focal neurological complaints. Physical examination: symmetrical, radial, ulnar, brachial, femoral, tibial, popliteal, carotid, and bilateral pulses, without murmurs, in addition to the absence of signs of joint hypermobility. Echocardiogram without changes. Doppler of renal arteries: increased blood flow in the middle third bilaterally. Laboratory tests such as phospholipids, antinuclear antibodies, antineutrophil antibodies, evidence of inflammatory activity, blood count, blood glucose, liver, kidney and thyroid function without changes. Excluding the probabilities of collagenosis, vasculitis, infections and hypermobility conditions, brain injuries in vertebral arteries, early hypertension in a young patient with a low body mass index and increased blood flow on renal Doppler justify the diagnosis of FMD, indicating referral of a vascular surgeon for follow-up.

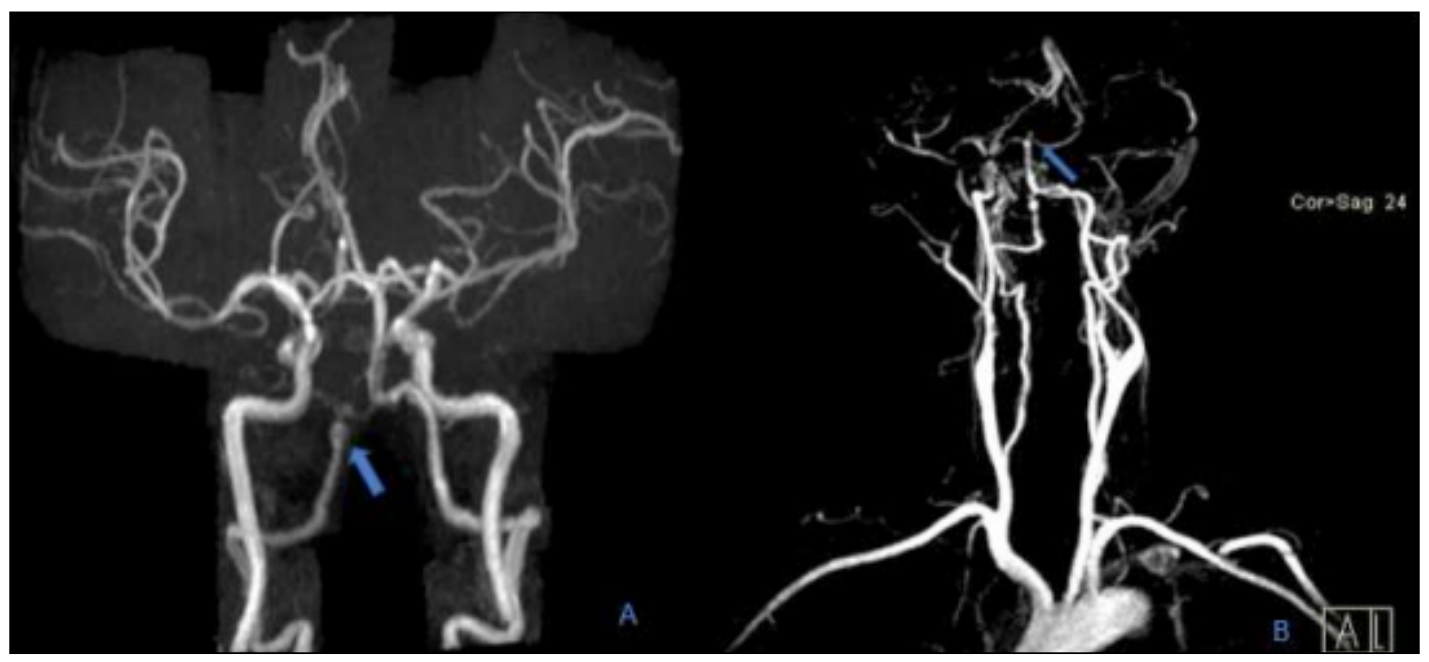

Figure 1. Focal area of dilation as demonstrated magnetic angioresonance. 


\section{CONCLUSION}

The FMD diagnosis is complex and little suspected in medical practice, often due to atypical or discrete clinical findings and difficulty in vascular histopathology. Imaging exams are useful for diagnosis and to rule out other hypotheses. The physician should be aware of secondary hypertension in patients without risk factors and more difficult to control and neurological changes (recent headache, focal changes, lowering the level of consciousness) for suspected preexisting vascular diseases and undiagnosed, such as FMD.

\section{KEYWORDS}

Vascular diseases, Fibromuscular dysplasia, Vasculitis. 